CLINICAL STUDY

\title{
The effect of GH replacement therapy on different fat compartments: a whole-body magnetic resonance imaging study
}

\author{
A Egger*, T Buehler ${ }^{1, *}$, C Boesch $^{1}$, P Diem, C Stettler and E R Christ \\ Division of Endocrinology, Diabetes, and Clinical Nutrition, Inselspital, Bern University Hospital and ${ }^{1}$ Department of Clinical Research, University of Bern, \\ CH-3010 Bern, Switzerland \\ (Correspondence should be addressed to E R Christ; Email: emanuel.christ@insel.ch)
}

*(A Egger and T Buehler contributed equally to this work)

\begin{abstract}
Objective: Patients with GH deficiency (GHD) are insulin resistant with an increase in visceral fat mass (FM). Whether this holds true when sedentary control subjects (CS) are matched for waist has not been documented.

GH replacement therapy (GHRT) results in a decrease in FM. Whether the decrease in FM is mainly related to a reduction in visceral FM remains to be proven. The aim was to separately assess visceral and subcutaneous FM in relation to insulin resistance (IR) in GHD patients before and after GHRT and in sedentary CS.

Methods: Ten patients with GHD were investigated before and 6 months after GHRT. Sedentary CS matched for age, gender, body mass index, and waist were assessed. Exercise capacity was measured as $\mathrm{VO}_{2 \max }$ using an incremental work load on a treadmill. Visceral and subcutaneous FM were measured using whole-body magnetic resonance imaging and IR by the homeostasis model assessment of IR (HOMA-IR) index.

Results: GHD patients had a non-significantly lower $\mathrm{VO}_{2 \max }$ but did not have increased subcutaneous and visceral FM compared with CS. GHRT resulted in a similar relative decrease in subcutaneous and visceral FM. Compared with CS, GHD patients showed a lower HOMA-IR. GHRT tended to increase HOMA-IR.

Conclusion: Matching for waist and separate assessment of visceral and subcutaneous FM may be critical in the evaluation of body composition and IR in GHD patients before and after GHRT.
\end{abstract}

European Journal of Endocrinology 164 23-29

\section{Introduction}

Hypopituitary patients with GH deficiency (GHD) present with altered body composition characterized by an increase in fat mass (FM), predominantly in the visceral compartment, and a decrease in fat-free mass (FFM) and total body water $(1,2)$. The increase in visceral FM in GHD patients has been linked to an insulin-resistant condition which in turn has been associated with an increased cardiovascular risk $(1,3)$. GH replacement therapy (GHRT) consistently results in a decrease in FM, and an increase in FFM and body water (1). Interestingly, there is data suggesting that GHRT reduces predominantly visceral FM (4). Shortterm GHRT leads usually to a limited increase in insulin resistance (IR) (5) whereas long-term supplementation tends to decrease IR possibly as a consequence of the increase in FFM (6).

A panoply of methods has been used to assess body composition in hypopituitarism with GHD before and after GHRT including bioimpedance (BIA) $(1,2,7,8)$, $40 \mathrm{~K}$ labeled water dilution technique $(7,9)$, computer tomography (CT) (4), and magnetic resonance imaging (MRI) (9) as well as the dual-energy X-ray absorptiometry (DEXA) (1, 7, 10-12). The 40K labeled water dilution technique and BIA assessment depend on total body water and/or electrolyte distribution, which have been shown to be affected by GHD and GHRT $(13,14)$. This may lead to an overestimation of changes in FFM using these methods $(7,15,16)$. DEXA scan does not offer the possibility to differentiate between subcutaneous and visceral FM, a distinction which may be of particular clinical importance in these patients.

The number of methods that reliably determine visceral FM is currently limited. Recent data suggest that abdominal cross-sectional imaging (MRI or CT) is less reliable than whole abdomen scanning in determining visceral FM $(16,17)$. Furthermore, whole-body MRI has been established for the separate assessment of the different body fat compartments (total, subcutaneous, 
and visceral FM) in different scientific settings, including insulin-resistant conditions (18).

In this study, we measured the different fat compartments in hypopituitary patients with GHD (before and after GHRT) and in sedentary control subjects (CS) matched for gender, age, body mass index (BMI), and waist. We hypothesized that GHD patients have predominantly an increase in visceral FM compared with matched CS that will be reduced following GHRT.

\section{Subjects and methods}

The study was a prospective single-center open casecontrol study. It was performed at the University Hospital of Bern, Switzerland. Investigations were done at the Division of Endocrinology, Diabetes and Clinical Nutrition and at the Department of Clinical Research of the University of Bern.

Four women and six men with severe GHD due to pituitary or hypothalamic disease were recruited from the outpatient clinic. GHD was defined as a peak GH of $<3 \mathrm{mU} / \mathrm{l}$ during an insulin tolerance test with nadir plasma glucose of $<2.2 \mathrm{mmol} / \mathrm{l}$ and hypoglycemic symptoms (19). Patients were included, provided they had been under stable conventional hormone replacement therapy (glucocorticoids, thyroxine $\left(\mathrm{T}_{4}\right)$, and sex hormones) as needed for at least 6 months. Exclusion criteria were (former or present) ACTH- or GH-secreting pituitary adenoma, abnormal liver or renal function, active neoplasia, severe cardiovascular disease (unstable coronary artery disease, heart failure New York Heart Association III-IV), diabetes mellitus, hemophilia, therapy with drugs known to affect lipid or glucose metabolism, inability to exercise and contraindications to exposure to a 3-Tesla magnetic field. Additionally, we recruited ten sedentary CS matched for age, gender, BMI, and waist.

The study was performed according to the Declaration of Helsinki, the guidelines of good clinical practice, the Swiss health laws, and the ordinance on clinical research. Approval for the study was obtained from the ethics committee, Bern. Each study subject gave written informed consent.

\section{Study protocol}

GHD patients and CS attended the hospital after an overnight fast. In GHD patients with LH/FSH insufficiency, the investigation of body composition was performed in the middle of the dose interval in male patients and during replacement therapy in female patients respectively. In patients with TSH insufficiency, the dose was adjusted to obtain stable free $\mathrm{T}_{4}$ concentrations in the upper half of the normal range. Body weight was measured on an electronic balance with subjects wearing light clothes and without shoes. Height was assessed by a stadiometer. BMI was calculated as the weight divided by the square of the height. The waist circumference was measured in the upright position with a flexible tape placed on a horizontal plane at the level of the iliac crest. The measurement was made at the end of a normal expiration. Fasting baseline blood sample was collected; body composition was determined by BIA and immediately afterward by whole-body MRI in a 3-Tesla wholebody system. On a separate visit, a spiroergometry was carried out in all patients and sedentary CS using an incremental workload on a treadmill with the determination of peak aerobic capacity $\left(\mathrm{VO}_{2 \max }\right)$.

Patients with GHD were instructed about selfadministration of GH using a pen device (GenotropinPen, Pfizer, Switzerland). Usual clinical care was provided (monthly visit with insulin-like growth factor 1 (IGF1) measurements to adjust GH doses). GH dose was gradually increased to obtain IGF1 concentrations in the upper half of the age-adjusted reference range as suggested by the GH Research Society (19). After 6 months of GHRT, weight maintaining diet (total need of calories/d calculated according to the formula of HarrisBenedict, qualitatively consisting of $50 \%$ carbohydrate, $30 \%$ fat, and $20 \%$ protein), and identical physical activity, the same studies were performed in the patients.

\section{Whole-body fat analysis}

Images were acquired on a 3T MR system (Tim Trio; SIEMENS, Erlangen, Germany) with the body coil as combined transmit/receive coil. To determine visceral FM, subcutaneous FM, and whole-body volume (WBV), images were taken in axial direction with a T1-weighted fast spin echo technique $(\mathrm{TR}=452 \mathrm{~ms}, \mathrm{TE}=38 \mathrm{~ms}$, echo train length $=7$, slice thickness of $10 \mathrm{~mm}$, five slices per sequence, spacing between slices $20 \mathrm{~mm}$, FOV $50 \mathrm{~cm}$, image resolution $2 \mathrm{~mm}$ per pixel) from fingers to toes leading to 100-130 axial images per subject. Owing to the limited displacement of the examination table, the subjects were measured in two positions, prone/head-first with outstretched arms and prone/ feet-first. A mark was set at the iliac crest to reposition the subject. In the abdominal region, the slices were recorded in breath hold.

Image analysis for volume determination was done by using a home-built program (MATLAB R2007a, The MathWorks, Natick, MA, USA) that is based on an extended point counting method (20) and three sequential steps for the determination of visceral FM and subcutaneous FM: i) the region of visceral fat is separated from subcutaneous fat by a simple contour line, ii) the points for the point counting method are set or deleted by the program based on a threshold value, and iii) visual inspection of the points lets the operator correct for intensity variations resulting from radio frequency (RF) inhomogeneity. For the WBV, no separation by the contour line was applied, and the threshold was set to a minimal value 
(above noise level). Thus, except the volume of the lung, all tissues were counted for WBV.

The precision of the volumetric technique had been established in a phantom model with up to eight bottles filled with a known amount of oil between bottles with water (21) (typically $1 \mathrm{l}$ per bottle). Aluminum foil between the bottles generated highly inhomogeneous images, which corresponded to the complexity and inhomogeneity of real abdominal images at higher magnetic field strength. The images were analyzed by three observers twice. The Bland-Altman analysis of the comparison between measured and true volumes resulted in a coefficient of variance $(\mathrm{CV})$ of $4.5 \%$, the comparison between the first and second determination by an observer (intra-observer variability) resulted in a CV of $4.1 \%$, and the comparison between the values determined by the three observers (inter-observer variability) resulted in a $\mathrm{CV}$ of $4.2 \%$.

For the study, the analysis of the images was performed at the end of the study by a single operator who was not part of the clinical team, and completely blinded to the group of the volunteers or the treatment status of the patients.

To estimate the accuracy of the WBV, the weight of the subjects was calculated based on a two-compartment model with a fat compartment (density for adipose tissue $0.918 \mathrm{~g} / \mathrm{ml},(22)$ ) and an FFM (average density for the FFM $1.100 \mathrm{~g} / \mathrm{ml}(23))$.

\section{Biochemical analysis}

Serum IGF1 was measured by an IRMA (Nichols Institute, San Juan Capistrano, CA, USA), and plasma glucose was measured using the hexokinase method (Hitachi 917, Roche). Insulin was determined by RIA (Linco Research, Labodia, Yens, Switzerland).

\section{Statistical analysis}

Statistical analysis was performed in SPSS 17.0 (SPSS, Inc., Chicago, IL, USA). Results are expressed as median \pm interquartile range. Non-parametric Wilcoxon signed-rank test was used to compare unpaired datasets (GHD versus CS), whereas nonparametric Mann-Whitney tests were used to compare paired data (GHD versus GHRT). A Pearson correlation was calculated to compare body weight based on MRI assessment with body weight measured on a scale. A two-sided $P$ value of $<0.05$ was considered significant.

\section{Results \\ Clinical and biochemical findings}

Individual clinical characteristics of the GHD patients are summarized in Table 1 . At the end of the study, one of the GHD patients had to be withdrawn due to a discus hernia with a need for a surgical intervention, which was judged not to be related to GHRT (Tables 1-3).

The final dose of $\mathrm{GH}$ was attained at 3 months. During the remaining 3 months, a stable GH dose was administered. The median dose was $0.5 \mathrm{mg} /$ day in male patients (range $0.3-0.6 \mathrm{mg} /$ day) and $0.7 \mathrm{mg} /$ day (range $0.5-1.2 \mathrm{mg} /$ day) in female patients.

All except one patient had LH/FSH insufficiency. All the male patients $(n=6)$ were substituted with testosterone undecanoas $1000 \mathrm{mg}$ every $10-12$ weeks. Two of the female patients with LH/FSH insufficiency were postmenopausal. One of the postmenopausal patients was substituted with a combination including estradiol valeras and cyproteron acetas, one premenopausal female patient was treated with a anticonceptive combination therapy including gestodenum $0.075 \mathrm{mg}$

Table 1 Clinical characteristics of patients presenting with GHD.

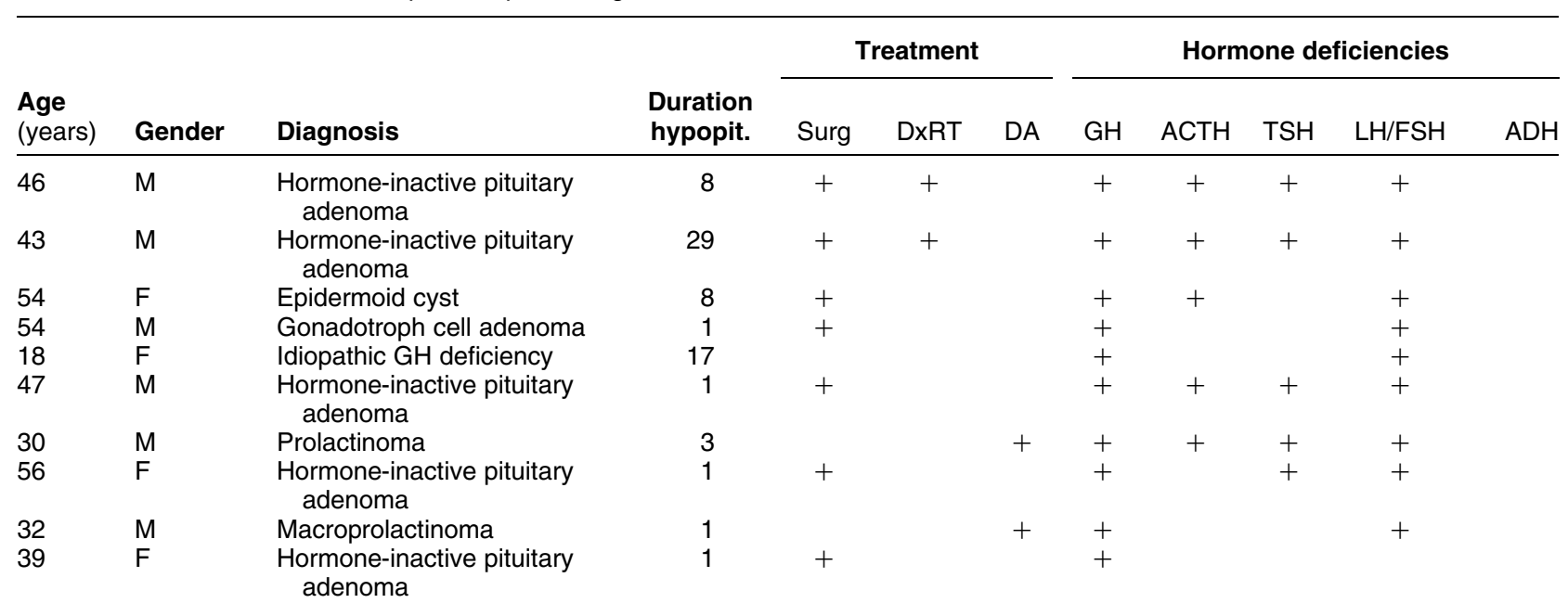

Hypopit., hypopituitarism; Surg, surgery; DxRT, pituitary radiotherapy; DA, dopamine agonist; ADH, antidiuretic hormone. 
Table 2 Anthropometric, biochemical, and body composition characteristics of GHD before and after therapy (GHRT).

\begin{tabular}{lccc}
\hline & GHD & GHRT & P values \\
\hline Males/females & $6 / 4$ & $6 / 4$ & \\
Age (years) & $45.5(32.0-54.6)$ & $46.1(32.6-55.1)$ & $\mathbf{0 . 0 0 5}$ \\
${\text { Waist }(\mathrm{cm})^{\mathrm{a}}}_{\mathrm{BMI}\left(\mathrm{kg} / \mathrm{m}^{2}\right)}$ & $90.5(78.9-96.9)$ & $91.0(77.5-93.5)$ & 0.362 \\
Baseline IGF1 (ng/ml) & $27.2(22.9-29.0)$ & $27.3(22.3-29.8)$ & 0.575 \\
HOMA-IR & $68.5(40.3-87.6)$ & $153.0(112.0-174.3)$ & $\mathbf{0 . 0 0 5}$ \\
FM BIA (kg) & $0.74(0.31-1.65)$ & $1.14(0.62-2.10)$ & 0.169 \\
FFM BIA (kg) & $21.4(17.0-28.4)$ & $19.7(14.5-25.6)$ & $\mathbf{0 . 0 4 7}$ \\
Total FM MRI (kg) $^{\mathrm{a}}$ & $51.6(43.7-60.0)$ & $54.0(44.4-65.7)$ & $\mathbf{0 . 0 0 5}$ \\
Subcutaneous FM MRI (kg) $^{\mathrm{a}}$ & $19.0(14.7-25.5)$ & $17.6(12.0-24.3)$ & $\mathbf{0 . 0 0 8}$ \\
Visceral FM MRI (kg) $^{\mathrm{a}}$ & $16.0(13.8-22.7)$ & $14.7(10.8-21.6)$ & $\mathbf{0 . 0 0 8}$ \\
FFM MRI (kg) $^{\mathrm{a}}$ & $2.1(1.3-3.1)$ & $2.0(1.1-2.6)$ & $\mathbf{0 . 0 4 4}$ \\
\hline
\end{tabular}

Results are median and interquartile range. Bold $P$ values, statistically significant.

${ }^{a}$ Analysis based on nine paired datasets.

and ethinylestradiol $0.02 \mathrm{mg}$ replacement therapy. $\mathrm{T}_{4}$ replacement therapy was necessary in half of the patients. The dose ranged between 0.075 and 0.175 (median $0.1 \mathrm{mg}$ ). Cortisol replacement therapy was initiated in half of the patients. The dose of cortisol was adapted to the body weight and administered in two or three doses as done previously (24). The cortisol replacement dose used in the GHD patients was between 15 and $20 \mathrm{mg}$ (median $17.5 \mathrm{mg} /$ day).

Physical fitness as assessed by the determination of $\mathrm{VO}_{2 \max }$ was non-significantly lower in the GHD patients than in the CS (GHD: 37.1 (31.5-40.2) versus CS: 39.9 (38.1-45.9) $\mathrm{ml} \mathrm{O}_{2} / \mathrm{kg}$ body weight; $P=0.10$, median and interquartile range).

Additional anthropometric and fasting biochemical results of the GHD patients and CS are shown in Tables 2 and 3.

GHD patients and CS did not differ regarding the matching criteria: gender, age, BMI, and waist. GHRT did not have a significant influence on neither weight, BMI nor waist (Table 2).
GHD patients had significantly lower IGF1 levels than CS. GHRT led to a significant increase in IGF1 levels.

GHD patients showed a lower homeostasis model assessment of IR (HOMA-IR) than CS. GHRT tended to increase HOMA-IR, but the difference did not reach conventional levels of statistical significance.

\section{Bioimpedance}

Results of BIA are summarized in Tables 2 and 3. GHD patients and CS did not differ regarding FM and FFM. GHRT lead to a significant decrease in FM $(P<0.047)$ in parallel with a significant increase in FFM $(P<0.005)$.

\section{Whole-body MRI}

Table 3 and Fig. 1 summarize the results of body composition as assessed by whole-body MRI (Tables 2 and 3 and Fig. 1). GHD patients and CS did not significantly differ with regard to the amount of total

Table 3 Baseline anthropometric, biochemical, and body composition characteristics of GHD patients and matched sedentary controls (CS).

\begin{tabular}{lccc}
\hline & GHD & CS & $\begin{array}{c}\boldsymbol{P} \text { values } \\
\text { (CS vs GHD) }\end{array}$ \\
\hline Males/females & $6 / 4$ & $6 / 4$ & \\
Age (years) & $45.5(32.0-54.6)$ & $44.4(30.6-53.6)$ & 0.940 \\
Waist (cm) & $91.6(82.3-100.3)$ & $90.0(80.5-97.9)$ & 0.821 \\
BMI (kg/m²) & $27.2(22.9-29.0)$ & $23.9(22.0-29.2)$ & 0.450 \\
Baseline IGF1 (ng/ml) & $68.5(40.3-87.6)$ & $111.5(93.8-138.3)$ & 0.001 \\
HOMA-IR & $0.74(0.31-1.65)$ & $1.42(1.17-2.34)$ & 0.034 \\
FM BIA (kg) & $21.4(17.0-28.4)$ & $16.6(15.4-30.0)$ & 0.496 \\
FFM BIA (kg) & $51.6(43.7-60.0)$ & $51.7(40.5-64.0)$ & 0.970 \\
Total FM MRI (kg) & $19.2(14.8-24.8)$ & $18.1(13.6-25.3)$ & 0.650 \\
Subcutaneous FM MRI (kg) & $16.4(14.0-22.1)$ & $15.2(12.1-23.4)$ & 0.545 \\
Visceral FM MRI (kg) $^{\mathrm{a}}$ & $2.4(1.5-3.3)$ & $2.6(1.3-3.4)$ & 0.940 \\
FFM MRI (kg) $^{\mathrm{a}}$ & $51.1(43.6-62.8)$ & $50.9(39.3-65.1)$ & 1.000 \\
\hline
\end{tabular}

Results are median and interquartile range. Bold $P$ values, statistically significant.

${ }^{a}$ Differences between median values of GHD patients by the inclusion of ten patients as compared with Table 2 . 

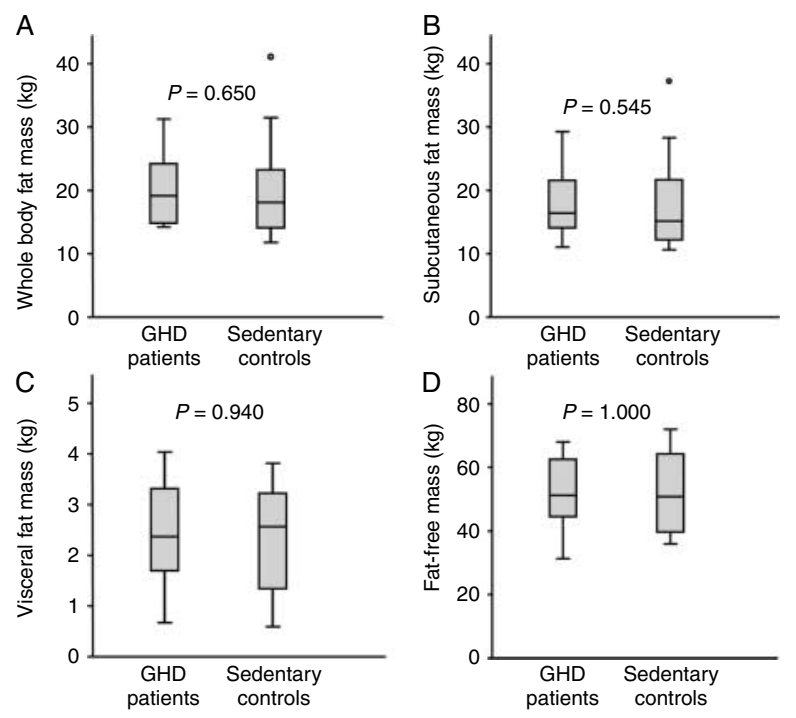

Figure 1 Comparison of body composition of GHD patients and matched sedentary control subjects. (A) Whole-body fat mass, (B) subcutaneous fat mass, (C) visceral fat mass, and (D) fat-free mass. Interquartile ranges are plotted according to Tukey's Hinges (SPSS 17.0). $P$ values are shown for the comparison against GHD patients.

body FM, subcutaneous FM, visceral FM, and FFM (Table 3 and Fig. 1A-D).

GHRT led to a significant decrease in total body FM, subcutaneous FM, and visceral FM. The relative decrease in visceral FM (17\%) and subcutaneous FM $(12 \%)$ was similar. In addition, a significant increase in FFM was observed.

\section{Correlations}

There was an excellent correlation between the weight calculated from MRI and weight measured on a scale (body weight by scale $=1.032 *$ weight by MRI $-0.043 \mathrm{~kg}$; $\left.R^{2}=0.98 ; P<0.001\right)$. The absolute calculated body weight based on the MRI method resulted in a mean underestimation of body weight of $2.3 \pm 1.9 \mathrm{~kg}$ (mean \pm s.D.).

\section{Discussion}

The main results of this study are twofold: i) hypopituitary patients with GHD do not have an increase in subcutaneous and visceral FM compared with CS matched for age, gender as well as for BMI and waist, ii) GHRT reduced both subcutaneous and visceral FM to a similar extent.

Using whole-body MRI, total FM and its distribution in the subcutaneous and visceral compartments were not significantly different between GHD patients and sedentary CS. This is in contrast to previous studies (1) and our hypothesis. A possible explanation for this controversial finding may be the fact that this study included waist circumference as an additional criteria to match the GHD patients. Furthermore, in contrast to previous studies $(4,25)$, half of our GHD patients had a sufficient corticotrophin axis (Table 1) as assessed by an insulin tolerance test, and the remaining were adequately substituted with hydrocortisone (twice or thrice daily regime) according to a weight-adapted dose regime (24). In this study, the median substitution dose of hydrocortisone was $17.5 \mathrm{mg} /$ day $(0.20 \mathrm{mg} / \mathrm{kg}$ body weight), significantly lower than in previous studies where daily doses between 25 and $30 \mathrm{mg}$ were reported $(4,25)$. The fact that the dose of hydrocortisone replacement therapy may have a significant impact on body FM, in particular on visceral FM, is further substantiated by a recent study that reports a significant reduction in total and abdominal FM following a decrease in dose of hydrocortisone from 20-30 to 10-15 mg/day in hypopituitary patients (26).

HOMA-IR scores indicate that GHD patients are unexpectedly less resistant than the sedentary CS. Using different methods of IR measurements, previous studies suggest that GHD patients exhibit an increase in IR compared with CS matched for age, gender, and BMI $(27,28)$. Based on the small sample size, the current findings have to be interpreted with caution. However, it is tempting to speculate that in the presence of a similar waist circumference and a comparable amount of visceral FM, GHD patients show higher insulin sensitivity due to a lack of $\mathrm{GH}$ than CS. Consequently, the reported increase in IR of GHD patients observed in previous studies may rather be a consequence of the choice of CS than the GHD status of the patients. Alternatively, the higher hydrocortisone replacement dose usually administered in previous studies may have been an additional confounding factor. Differences in duration of GHD are unlikely to contribute to these controversial results since the mean duration of hypopituitarism in this study was similar than in previous studies ( $7 \pm 9$ years) (1). Similarly, differences in physical fitness do not explain these findings since GHD patients presented with slightly lower $\mathrm{VO}_{2 \max }$ than the matched sedentary CS.

GHRT resulted in a significant decrease in total, visceral, and subcutaneous FM and an increase in FFM. Quantitatively, the changes in total FM and FFM were consistent with previous studies (1). Moreover, in keeping with preceding reports (29), HOMA-IR index tended to increase following GHRT, albeit not reaching the levels of statistical significance. Interestingly, the relative decrease in subcutaneous FM associated with GHRT was accompanied by a comparable relative decrease in visceral FM. This is consistent with the fact that GH receptors are expressed at a quantitatively similar level in subcutaneous and visceral FM suggesting a comparable lipolytic action of GH at both sites (30). In contrast, previous studies proposed a predominant decrease in visceral FM following GHRT (4). Whether the predominant effect on visceral 
FM in the preceding studies may at least partly be explained by the established effect of $\mathrm{GH}$ on 11- $\beta$-hydroxysteroid dehydrogenase type-1 (31), which is likely to be of particular importance in the presence of high substitution dose of cortisone acetate (not used in this study), remains speculative.

The comparison of the weight based on the measured value on a scale and the calculated weight based on the whole-body MRI scan yielded an excellent correlation in the different study populations. However, a slight underestimation of the weight of about $2 \mathrm{~kg}$ by MRI scanning was observed. This is most likely due to an inconsistent differentiation of certain tissues such as hilus/lung tissue or air in the abdomen and the fact that the GHD patients and CS were weighted on a scale wearing light clothes. Since the aim of the comparison between MRI volume-based body weight estimation and body weight measured by a scale served as an indicator of the feasibility of the method and was not a primary physiological target, no extra steps were taken into consideration to further reduce this difference.

Our study has certain limitations. The number of participants was relatively small due to the additional matching criteria 'waist' of the CS. Further studies with more patients and CS are warranted to draw firm conclusions. In addition, we did not compare the wholebody FM assessment using whole-body MRI with DEXA, which is the gold standard for the assessment of body composition in endocrine diseases. However, we found an excellent correlation of MR results with the body weight measured on the scale. Furthermore, DEXA scanning is essentially based on the measurement of body composition in two dimension, whereas whole-body MRI scanning allows to assess it in three dimension. Finally, the method is capable of detecting GHRT-induced changes in body composition that are quantitatively comparable to previous results observed using $\operatorname{DEXA}(7,11,12)$.

In conclusion, this study indicates that GHD patients do not have an increase in visceral FM compared with CS matched for age, gender as well as for BMI and waist. GHRT results in a comparable relative reduction in subcutaneous and visceral FM. Matching for waist and separate assessment of visceral and subcutaneous FM may be critical in the evaluation of body composition and IR in GHD patients before and after GHRT.

\section{Declaration of interest}

The authors declare that there is no conflict of interest that could be perceived as prejudicing the impartiality of the research reported.

\section{Funding}

The work was funded by grants from the Swiss National Foundation to E R Christ (No. \#32000B0-100146) and C Boesch (No. \#310000118219) and by the Independent Pfizer Research Grant (to E R Christ). C Stettler is a PROSPER fellow supported by the Swiss National Science Foundation (Grant Number 3233B0 115212). Pfizer AG Switzerland kindly provided GH. We cordially thank Regula und Sabine Koenig for technical assistance.

\section{Acknowledgements}

We thank all the enthusiastic patients and control subjects who agreed to participate in this study.

\section{References}

1 Carroll PV, Christ ER, Bengtsson BA, Carlsson L, Christiansen JS, Clemmons D, Hintz R, Ho K, Laron Z, Sizonenko P, Sonksen PH, Tanaka T \& Thorne M. Growth hormone deficiency in adulthood and the effects of growth hormone replacement: a review. Growth Hormone Research Society Scientific Committee. Journal of Clinical Endocrinology and Metabolism 199883 382-395. (doi:10.1210/jc. 83.2.382)

2 Amato G, Mazziotti G, Di Somma C, Lalli E, De Felice G, Conte M, Rotondi M, Pietrosante M, Lombardi G, Bellastella A, Carella C \& Colao A. Recombinant growth hormone $(\mathrm{GH})$ therapy in GH-deficient adults: a long-term controlled study on daily versus thrice weekly injections. Journal of Clinical Endocrinology and Metabolism 200085 3720-3725. (doi:10.1210/jc.85.10.3720)

3 Bengtsson BA. Untreated growth hormone deficiency explains premature mortality in patients with hypopituitarism. Growth Hormone \& IGF Research 19988 (Supplement A) 77-80. (doi:10. 1016/S1096-6374(98)80014-2)

4 Bengtsson BA, Eden S, Lonn L, Kvist H, Stokland A, Lindstedt G, Bosaeus I, Tolli J, Sjostrom L \& Isaksson OG. Treatment of adults with growth hormone (GH) deficiency with recombinant human GH. Journal of Clinical Endocrinology and Metabolism 1993 76 309-317. (doi:10.1210/jc.76.2.309)

5 Fowelin J, Attvall S, Lager I \& Bengtsson BA. Effects of treatment with recombinant human growth hormone on insulin sensitivity and glucose metabolism in adults with growth hormone deficiency. Metabolism $1993 \mathbf{4 2}$ 1443-1447. (doi:10.1016/ 0026-0495(93)90197-V)

6 Johannsson G, Marin P, Lonn L, Ottosson M, Stenlof K, Bjorntorp P, Sjostrom L \& Bengtsson BA. Growth hormone treatment of abdominally obese men reduces abdominal fat mass, improves glucose and lipoprotein metabolism, and reduces diastolic blood pressure. Journal of Clinical Endocrinology and Metabolism 199782 727-734. (doi:10.1210/jc.82.3.727)

7 Koranyi J, Bosaeus I, Alpsten M, Bengtsson BA \& Johannsson G. Body composition during GH replacement in adults - methodological variations with respect to gender. European Journal of Endocrinology 2006154 545-553. (doi:10.1530/eje.1.02118)

8 Christ ER, Cummings MH, Westwood NB, Sawyer BM, Pearson TC, Sonksen PH \& Russell-Jones DL. The importance of growth hormone in the regulation of erythropoiesis, red cell mass, and plasma volume in adults with growth hormone deficiency. Journal of Clinical Endocrinology and Metabolism $1997 \mathbf{8 2}$ 2985-2990. (doi:10.1210/jc.82.9.2985)

9 Snel YE, Doerga ME, Brummer RM, Zelissen PM \& Koppeschaar HP. Magnetic resonance imaging-assessed adipose tissue and serum lipid and insulin concentrations in growth hormone-deficient adults. Effect of growth hormone replacement. Arteriosclerosis, Thrombosis, and Vascular Biology 199515 1543-1548.

10 Attanasio AF, Howell S, Bates PC, Frewer P, Chipman J, Blum WF \& Shalet SM. Body composition, IGF-I and IGFBP-3 concentrations as outcome measures in severely GH-deficient (GHD) patients after childhood GH treatment: a comparison with adult onset GHD patients. Journal of Clinical Endocrinology and Metabolism 200287 3368-3372. (doi:10.1210/jc.87.7.3368)

11 Attanasio AF, Shavrikova E, Blum WF, Cromer M, Child CJ, Paskova M, Lebl J, Chipman JJ \& Shalet SM. Continued growth hormone $(\mathrm{GH})$ treatment after final height is necessary to complete somatic development in childhood-onset GH-deficient patients. Journal of Clinical Endocrinology and Metabolism $2004 \mathbf{8 9}$ 4857-4862. (doi:10.1210/jc.2004-0551)

12 Weaver JU, Monson JP, Noonan K, John WG, Edwards A, Evans KA \& Cunningham J. The effect of low dose recombinant 
human growth hormone replacement on regional fat distribution, insulin sensitivity, and cardiovascular risk factors in hypopituitary adults. Journal of Clinical Endocrinology and Metabolism 1995 80 153-159. (doi:10.1210/jc.80.1.153)

13 Moller J, Frandsen E, Fisker S, Jorgensen JO \& Christiansen JS. Decreased plasma and extracellular volume in growth hormone deficient adults and the acute and prolonged effects of $\mathrm{GH}$ administration: a controlled experimental study. Clinical Endocrinology 199644 533-539. (doi:10.1046/j.1365-2265.1996.728550.x)

14 Salomon F, Cuneo RC, Hesp R \& Sonksen PH. The effects of treatment with recombinant human growth hormone on body composition and metabolism in adults with growth hormone deficiency. New England Journal of Medicine 1989321 1797-1803. (doi:10.1056/NEJM198912283212605)

15 Haymond MW, Sunehag AL \& Ellis KJ. Body composition as a clinical endpoint in the treatment of growth hormone deficiency. Hormone Research 199951 (Supplement 3) 132-140. (doi:10. 1159/000053176)

16 Ludescher B, Machann J, Eschweiler GW, Vanhofen S, Maenz C, Thamer C, Claussen CD \& Schick F. Correlation of fat distribution in whole body MRI with generally used anthropometric data. Investigative Radiology $2009 \mathbf{4 4}$ 712-719. (doi:10.1097/RLI. Ob013e3181afbb1e)

17 Thomas EL, Saeed N, Hajnal JV, Brynes A, Goldstone AP, Frost G \& Bell JD. Magnetic resonance imaging of total body fat. Journal of Applied Physiology $1998 \mathbf{8 5} 1778-1785$.

18 Machann J, Thamer C, Schnoedt B, Haap M, Haring HU, Claussen CD, Stumvoll M, Fritsche A \& Schick F. Standardized assessment of whole body adipose tissue topography by MRI. Journal of Magnetic Resonance Imaging 200521 455-462. (doi:10. 1002/jmri.20292)

19 Sonksen $\mathrm{PH} \&$ Christiansen JS. Consensus guidelines for the diagnosis and treatment of adults with growth hormone deficiency. Growth Hormone Research Society. Growth Hormone E IGF Research 19988 (Supplement B) 89-92. (doi:10.1016/ S1096-6374(98)80028-2)

20 Buehler TRN, Machann J, Schwenzer N \& Boesch C. Determination of body compartments at 1.5 and 3 Tesla combining three volume estimation methods. Proceedings of the International Society for Magnetic Resonance in Medicine 2010182915.

21 Buehler TCD \& Boesch C. Determination of whole body fat and visceral adipose tissue, combining three volume estimation methods. Proceedings of the International Society for Magnetic Resonance in Medicine 2009172872.
22 Boesch C, Machann J, Vermathen P \& Schick F. Role of proton MR for the study of muscle lipid metabolism. NMR in Biomedicine 2006 19 968-988. (doi:10.1002/nbm.1096)

23 Visser M, Gallagher D, Deurenberg P, Wang J, Pierson RN Jr \& Heymsfield SB. Density of fat-free body mass: relationship with race, age, and level of body fatness. American Journal of Physiology 1997272 E781-E787.

24 Mah PM, Jenkins RC, Rostami-Hodjegan A, Newell-Price J, Doane A, Ibbotson V, Tucker GT \& Ross RJ. Weight-related dosing, timing and monitoring hydrocortisone replacement therapy in patients with adrenal insufficiency. Clinical Endocrinology 200461 367-375. (doi:10.1111/j.1365-2265.2004.02106.x)

25 Snel YE, Brummer RJ, Doerga ME, Zelissen PM, Bakker CJ, Hendriks MJ \& Koppeschaar HP. Adipose tissue assessed by magnetic resonance imaging in growth hormone-deficient adults: the effect of growth hormone replacement and a comparison with control subjects. American Journal of Clinical Nutrition 199561 1290-1294.

26 Danilowicz K, Bruno OD, Manavela M, Gomez RM \& Barkan A. Correction of cortisol overreplacement ameliorates morbidities in patients with hypopituitarism: a pilot study. Pituitary 200811 279-285. (doi:10.1007/s11102-008-0126-2)

27 Johannsson G \& Bengtsson BA. Growth hormone and the metabolic syndrome. Journal of Endocrinological Investigation 199922 41-46.

28 Jorgensen JO, Krag M, Jessen N, Norrelund H, Vestergaard ET, Moller $\mathrm{N} \&$ Christiansen JS. Growth hormone and glucose homeostasis. Hormone Research 200462 (Supplement 3) 51-55. (doi:10.1159/000080499)

29 Fowelin J, Attvall S, von Schenck H, Bengtsson BA, Smith U \& Lager I. Effect of prolonged hyperglycemia on growth hormone levels and insulin sensitivity in insulin-dependent diabetes mellitus. Metabolism $1993 \mathbf{4 2}$ 387-394. (doi:10.1016/00260495(93)90092-3)

30 Fisker S, Hansen B, Fuglsang J, Kristensen K, Ovesen P, Orskov H \& Jorgensen JO. Gene expression of the GH receptor in subcutaneous and intraabdominal fat in healthy females: relationship to GH-binding protein. European Journal of Endocrinology 2004150 773-777. (doi:10.1530/eje.0.1500773)

31 Agha A \& Monson JP. Modulation of glucocorticoid metabolism by the growth hormone - IGF-1 axis. Clinical Endocrinology 200766 459-465. (doi:10.1111/j.1365-2265.2007.02763.x)

Received 17 September 2010

Accepted 7 October 2010 